\title{
Biscoitos enriquecidos com farelo de linhaça marrom (Linum usitatissiumun L.): valor nutritivo e aceitabilidade
}

\section{Biscuits enriched with brown flaxseed (LinumustatissiumnL.): nutritional value and acceptability}

Camila Coutinho de Moura'

Nathalia Peter'

Bianca de Oliveira Schumacker'

Lúcia Rota Borges

Elizabete Helbig'

' Departamento de Nutrição, Faculdade de Nutrição. Universidade Federal de Pelotas. Pelotas, RS, Brasil.

Apoio Financeiro: FAPERGS ARD

Processo n.: 10/0489-0

Correspondencia / Correspondence

Elizabete Helbig

E-mail: helbignt@gmail.com

\section{Resumo}

A incorporação de ingredientes a produtos de panificação tem crescido em função da preocupação com a saúde dos consumidores. A busca por uma alimentação saudável está cada vez mais presente na população, devido ao aumento da prevalência das doenças crônicas não transmissíveis. Em função disso, a indústria de alimentos tem desenvolvido produtos enriquecidos que de alguma forma possam contribuir com a saúde dos consumidores, evitando assim a ocorrência de doenças relacionadas à alimentação inadequada. A semente de linhaça marrom (Linum usitatissimum L.) é um ingrediente alimentar com potencial de uso em função de suas características benéficas, agregando valor nutricional aos alimentos. Com este estudo, objetivou-se formular biscoitos salgados com diferentes proporções de farelo de linhaça marrom e avaliar sua composição físico-química, cor e características sensoriais. Os biscoitos foram elaborados com a adição de diferentes concentrações de farelo de linhaça marrom, adição de 5\% (F5) e 20\% (F20), relacionados com o controle elaborado com farinha de trigo. Estes foram avaliados quanto a sua composição química, coloração, parâmetros físicos e aceitação sensorial. Os biscoitos elaborados com adição de farelo de linhaça marrom apresentaram maior teor de proteínas, resíduo mineral, fibras e umidade, comparados aos biscoitos elaborados com farinha de trigo sem adição de linhaça e coloração mais escura, e boa aceitação sensorial.

Palavras-chave: Linum usitatissimum. Alimento Functional. Linhaça. Valor Nutritivo. 


\section{Abstract}

The incorporation of ingredients to bakery products has grown due to consumers' concern with health. The search for a healthy diet is increasingly present in the population, due to increased prevalence of chronic noncommunicable diseases. As a result, food industry has developed products enriched in some way to contribute to the health of consumers, thus preventing the occurrence of diseases related to inadequate nutrition. The brown flaxseed (Linum usitatissimum L.) seed is a food ingredient with potential use due to its beneficial characteristics, adding nutritional value to food. This study aimed to formulate crackers with different proportions of bran, brown flaxseed and evaluate its physical and chemical composition, color and sensory characteristics. The cookies were prepared by adding different concentrations of brown flaxseed meal, adding $5 \%$ (F5) and 20\% (F20), related to the control prepared with wheat flour. These were evaluated for their chemical composition, color, physical parameters and sensory acceptance. Cookies prepared with added brown flaxseed bran had higher protein content, mineral residue, fiber and moisture, compared with darker cookies made with wheat flour without flaxseed, and good acceptability.

Key words: Linum usitatissimum. Functional Food. Flaxseed. Nutritive Value.

\section{Introdução}

A incorporação, nos alimentos, de substâncias com alegações funcionais tem crescido nos últimos anos por conta de suas propriedades benéficas para a saúde humana. ${ }^{1}$ Como exemplo, destaca-se a semente de linhaça (Linum usitatissimum L), uma oleaginosa em formato de grão oval, que pode ser encontrada nas colorações marrom ou dourada. Esse grão pode ser consumido in natura, triturado ou adicionado aos alimentos, sendo encontrado na forma de grão integral, moído e óleo. Seu aroma é semelhante ao das nozes, e tem sido incorporado a produtos, como pães, biscoitos e bolos. ${ }^{2,3} \mathrm{~A}$ linhaça marrom, oriunda de clima quente e úmido, também cultivada no Brasil, adapta-se muito bem ao nosso clima. ${ }^{4}$ 
Ao longo dos últimos anos, observa-se que o consumo de linhaça aumentou. Este fato tem sido atribuído ao conhecimento de seus efeitos na prevenção das doenças crônicas não transmissíveis (DCNT), associado ao estudo dos constituintes presentes no grão. Neste sentido, destacam-se os ácidos graxos essenciais, sendo que o ácido $\alpha$-linolênico (ômega 3) apresenta-se em maior quantidade, além de possuir fibras e proteínas. Esse grão é rico em lignanas, ácidos fenólicos, flavonóides, vitaminas e minerais. ${ }^{3,5}$

Por ser rica em ácido $\alpha$-linolênico, evidências sugerem que a linhaça apresenta efeitos hipocolesterolêmicos, atua na redução do LDL (lipoproteína de baixa densidade), e com isso previne doenças cardiovasculares. Estudos revelam que sua ingestão promove o aumento de HDL (lipoproteína de alta densidade) e suas propriedades antioxidantes atuam na prevenção do câncer e da aterosclerose. ${ }^{6-8}$ As fibras solúveis presentes na linhaça podem ajudar a reduzir o colesterol e controlar a glicemia, e as insolúveis auxiliam na digestão, reduzindo o trânsito intestinal e a constipação, e com isso podem ser úteis na prevenção do câncer. ${ }^{9}$

O farelo de linhaça é um notável antioxidante e imuno-estimulante, previne doenças degenerativas, cardiovasculares, e apresenta excelentes resultados no tratamento da tensão prémenstrual, menopausa e na redução dos riscos de câncer de mama, próstata e pulmão. ${ }^{10}$

A partir de evidências existentes em relação aos benefícios nutricionais da linhaça, ${ }^{11}$ pode-se peomover seu consumo por meio da incorporação em produtos alimentícios como, por exemplo, biscoitos.

O presente estudo tem como objetivos desenvolver uma formulação de biscoito salgado, acrescentando o farelo de linhaça marrom à farinha de trigo em diferentes proporções, e avaliar a composição físico-química, a cor e os parâmetros sensoriais desse biscoito.

\section{Metodologia}

Para a execução do estudo foram elaborados três tipos de biscoitos, C (Controle Trigo), F5 (farelo de linhaça marrom 5\%) e F20 (farelo de linhaça marrom 20\%), conforme descrito na tabela 1. 
Tabela 1. Formulação de biscoitos elaborados com farinha de trigo e biscoitos enriquecidos com farelo linhaça marrom (Linum usitatissiumun L.). Pelotas, RS, 2012.

\begin{tabular}{lccc}
\multicolumn{1}{c}{$\begin{array}{c}\text { Ingredientes } \\
\text { (gramas) }\end{array}$} & $\mathrm{C}$ & Tipos de Biscoitos & \\
\hline Farinha de trigo & 100 & $\mathrm{~F} 5$ & $\mathrm{~F} 20$ \\
Farelo de linhaça & 0 & 95 & 80 \\
Óleo de Soja & 16 & 18 & 20 \\
Sal & 3 & 3 & 3 \\
Fermento biológico & 1,7 & 1,70 & 1,70 \\
Bicarbonato de sódio & 0,20 & 0,20 & 0,20 \\
Tempero desidratado* & 0,50 & 0,50 & 0,50 \\
Água & $60 * *$ & $60 * *$ & $60 * *$ \\
\hline
\end{tabular}

*Salsa, alho, cebola, orégano

**A porcentagem dos ingredientes (com exceção da água) foi calculada em relação ao peso da farinha de trigo e linhaça marrom;

$\mathrm{C}=$ Controle (trigo); F5 = Farelo de linhaça marrom 5\%; F20 = Farelo de linhaça marrom 20\%

Para as análises químicas, os biscoitos foram triturados em moinho. Os conteúdos de cinzas, umidade e os teores de proteína bruta foram determinados de acordo com a Association of Official Analytical Chemists (AOAC). ${ }^{12}$ Lipídeos totais foram determinados pelo método descrito por Bligh \& Dyer. ${ }^{13}$ A quantidade de fibra bruta foi determinada conforme Angelucci et al. ${ }^{14}$ Os carboidratos foram calculados por diferença dos demais componentes.O valor calórico dos biscoitos foi calculado com base no valor calórico dos macronutrientes, sendo 4 kcal.g ${ }^{-1}$ para carboidratos e proteínas, e $9 \mathrm{kcal} . \mathrm{g}^{-1}$ para lipídios. As determinações foram realizadas em triplicata.

As análises físicas dos biscoitos foram determinadas de acordo com o método de 10-50D, da AOAC, ${ }^{12}$ para determinação da massa e diâmetro dos biscoitos antes e após o forneamento, espessura e fator de expansão. O volume específico foi calculado pela relação entre o volume aparente e peso do biscoito assado, sendo expresso em $\mathrm{g} \mathrm{e} \mathrm{cm}^{-1} \mathrm{O}$ perfil de textura dos biscoitos foi avaliado em texturômetro (texture analyser TA. XRplus, Stable Micro Systems).

A cor dos biscoitos foi determinada com utilização do colorímetro Minolta modelo CR-300, usando sistema CIEL*a*b*, no qual os valores de luminosidade (L*) variam de 0 (preto) e 100 (branco), e os valores das coordenadas de cromaticidade $a^{*}$ e b* variam de $-a^{*}$ (verde) até $+a^{*}$ (vermelho), e de $-b^{*}\left(\right.$ azul) até $+b^{*}$ (amarelo). 
Para a análise sensorial, o teste foi conduzido 24 horas após o forneamento dos biscoitos, com a participação de 60 julgadores não treinados, formados por professores, alunos e funcionários da Universidade Federal de Pelotas, abordados aleatoriamente, com idade entre 18 e 53 anos, que receberam as amostras de 4,0 \pm 0,5g, simultaneamente, servidas e codificadas com números de três dígitos, em blocos completos casualizados. Orientou-se que os provadores ingerissem água após cada degustação para limpeza do palato. Cada julgador recebeu três biscoitos, correspondentes a cada formulação, que foram avaliados quanto à aceitação sensorial, utilizando-se escala hedônica estruturada de nove pontos, que variou de "1" (desgostei extremamente) até "9" (gostei extremamente), conforme método descrito por Minim. ${ }^{15}$

Para a avaliação estatística, utilizou-se o teste de Tukey. O nível de significância considerado para os valores foi de $\mathrm{p}<0,05$.

O projeto foi aprovado pelo Comitê de Ética em Pesquisa da ESEF/UFPEL, protocolado sob número 015/2012.

\section{Resultados e discussão}

A composição química dos biscoitos elaborados com farinha de trigo enriquecidos com farelo de linhaça marrom está apresentada na tabela 2. Os biscoitos F5 e F20 apresentaram teores de umidade de 10,01\% e 10,90\%, respectivamente, e os valores de umidade estão em conformidade com os estipulados pela CNNPA, ${ }^{16}$ que deve ser menor que $14 \%$. De acordo com Teixeira et al., ${ }^{17}$ deve haver controle na umidade, pois esta interfere na qualidade física do produto, principalmente no parâmetro que avalia a dureza, pois quanto menor, a umidade maior é a dureza do biscoito.

Os biscoitos enriquecidos com farelo de linhaça marrom apresentaram maiores teores de proteínas, resíduo mineral e umidade, quando comparados aos biscoitos elaborados com farinha de trigo sem adição de farelo de linhaça marrom (tabela 2). Para as fibras, observou-se que o maior resultado foi obtido nos biscoitos formulados com $20 \%$ de farelo de linhaça marrom. Resultados semelhantes foram encontrados por Oliveira et al. ${ }^{18}$ na elaboração de pão utilizando farinha mista de trigo e linhaça.

O aumento do valor nutritivo nesses estudos é devido à composição do farelo de linhaça, $34,82 \%$ de fibra alimentar, $28 \%$ de proteína e 0,37\% de lipídeos, este em baixa quantidade por ter sido utilizado em nosso estudo um farelo desengordurado. Assim, o farelo de linhaça marrom apresenta valores maiores do que a farinha de trigo: 6,87\% de fibras, 13,44\% de proteínas e 1,73\% de lipídeos, de acordo com Heinemann et al. ${ }^{19}$.

Os biscoitos F20 apresentaram maior teor de fibra, com diferença significativa em relação ao controle e o F5 (tabela 2). Valores semelhantes foram encontrados por Borges et al., ${ }^{20}$ que 
avaliaram pão enriquecido com farinha integral de linhaça por meio de caracterização físicoquímica e sensorial.

Os percentuais de proteínas e cinzas aumentaram conforme a adição do farelo de linhaça, com diferença significativa entre si (tabela 2). O maior teor de resíduo mineral também foi encontrado por Hussain et al. ${ }^{9}$, quando adicionaram farinha de linhaça a cookies, e por Maciel, ${ }^{21}$ em estudo com adição de farinha de linhaça a biscoitos tipo cracker. Tal resultado se deve à presença de minerais na linhaça, pois esta possui maior teor de cinzas quando comparada à farinha de trigo especial, apresentando valores de $18,5 \mathrm{~g} / 100 \mathrm{~g}$ e $0,8 \mathrm{~g} / 100 \mathrm{~g}$, respectivamente.

Para os carboidratos, observou-se que F5 e F20 não diferiram entre si ( $\mathrm{p} \geq 0,05)$, mas diferiram significativamente quando comparadas ao controle (tabela 2).

Tabela 2. Composição centesimal dos biscoitos elaborados com farinha de trigo e com farelo de linhaça marrom em diferentes proporções (base seca). Pelotas, RS, 2012.

\section{Composição}

Tipos de Biscoitos

C F 5 F 20

\begin{tabular}{lccc}
\hline Proteína & $9,29 \pm 0,20^{\mathrm{c}}$ & $10,44 \pm 0,07^{\mathrm{b}}$ & $12,04 \pm 0,10^{\mathrm{a}}$ \\
Extrato Etéreo & $3,76 \pm 0,43^{\mathrm{a}}$ & $2,70 \pm 0,50^{\mathrm{a}}$ & $3,67 \pm 0,22^{\mathrm{a}}$ \\
Resíduo Mineral & $0,40 \pm 0,113^{\mathrm{c}}$ & $3,16 \pm 0,11^{\mathrm{b}}$ & $3,73 \pm 0,05^{\mathrm{a}}$ \\
Carboidrato & $87,79 \pm 2,30^{\mathrm{a}}$ & $83,70 \pm 0,43^{\mathrm{b}}$ & $80,55 \pm 0,26^{\mathrm{b}}$ \\
Fibra Total & $1,09 \pm 0,37^{\mathrm{b}}$ & $0,76 \pm 0,08^{\mathrm{b}}$ & $1,96 \pm 0,22^{\mathrm{a}}$ \\
Umidade & $9,75 \pm 0,03^{\mathrm{a}}$ & $10,01 \pm 0,21^{\mathrm{a}}$ & $10,90 \pm 0,04^{\mathrm{a}}$ \\
Valor calórico & $422,16^{\mathrm{a}}$ & $400,86^{\mathrm{a}}$ & $403,39^{\mathrm{a}}$ \\
(kcal.100g-1) & & & \\
\hline
\end{tabular}

*Letras diferentes na mesma linha, para o mesmo nutriente, indicam diferença significativa pelo teste de Tukey $(\mathrm{p}<0,05)$.

$* * \mathrm{C}=$ controle (trigo); F5 = farelo de linhaça 5\%; F20 = farelo de linhaça $20 \%$ 
Na tabela 3 estão apresentados os parâmetros de cor dos biscoitos, representados pelos valores de L* (luminosidade), a* e b* (cromaticidade). Todos os biscoitos elaborados com farelo de linhaça exibiram valores de $L^{*}$ e b* menores que o biscoito com farinha de trigo, ou seja, coloração mais escura. A coloração dos biscoitos está relacionada sobretudo com os ingredientes da formulação: biscoitos com maior teor de fibras costumam apresentar coloração mais escura. Resultados semelhantes foram apresentados por Alpaslan \& Hayta, ${ }^{22}$ ao utilizarem farinha de linhaça no processamento de pães.

Tabela 3. Parâmetros de cor dos biscoitos elaborados com farinha de trigo e com farelo de linhaça marrom em diferentes proporções. Pelotas, RS, 2012.

\begin{tabular}{cccc}
\hline Parâmetros de Cor & \multicolumn{3}{c}{ Tipos de Biscoitos } \\
\cline { 2 - 4 } & C & F5 & F20 \\
\hline L $^{*}$ & $67,57 \pm 2,85^{\mathrm{a}}$ & $59,32 \pm 1,47^{\mathrm{b}}$ & $42,56 \pm 2,13^{\mathrm{c}}$ \\
$\mathrm{a}^{*}$ & $1,29 \pm 0,13^{\mathrm{c}}$ & $2,97 \pm 0,25^{\mathrm{b}}$ & $5,96 \pm 0,48^{\mathrm{a}}$ \\
$\mathrm{b}^{*}$ & $23,79 \pm 0,95^{\mathrm{a}}$ & $21,01 \pm 1,80^{\mathrm{b}}$ & $16,75 \pm 1,35^{\mathrm{c}}$ \\
\hline
\end{tabular}

* Médias seguidas de letras diferentes nas colunas diferem entre si pelo teste de Tukey $(\mathrm{p}<0,05)$.

** C $=$ controle (trigo); F5 = farelo de linhaça 5\%; F20= farelo de linhaça 20\%

Na tabela 4 estão apresentados os parâmetros físicos, em que o peso antes e após o forneamento não apresentou diferença significativa entre as amostras.O fator de expansão foi maior para os biscoitos-controle e os enriquecidos com farelo de linhaça marrom 5\%, em relação aos biscoitos enriquecidos com farelo de linhaça marrom 20\% (tabela 4). O fator expansão está relacionado à capacidade dos ingredientes em absorver água, geralmente biscoitos com alto teor de fibras apresentam diminuição do fator de expansão, ${ }^{23}$ o que ocorreu em nosso estudo.

O volume específico variou entre $1,1 \mathrm{e} 1,28 \mathrm{~cm}^{3} \cdot \mathrm{g}^{-1}$, sendo que o menor valor encontrado foi para a amostra F20 (tabela 4). Nota-se que não houve diferença significativa entre as formulações com adição de farelo e o controle, e resultados semelhantes foram descritos por Oliveira et al. ${ }^{18}$.

A análise dos resultados de textura demonstrou valor menor de firmeza para a amostra F5 em relação à amostra C e F20 (tabela 4). A firmeza dos biscoitos está relacionada com a força aplicada para acarretar uma alteração ou rompimento da amostra, podendo ser relacionada com a mastigação humana. A força máxima avaliada para produtos panificados depende de inúmeros fatores, como qualidade da farinha, umidade da massa, conservação e quantidade de gorduras, açúcares, emulsificantes, enzimas, entre outros. ${ }^{24}$ Oliveira et al. ${ }^{18}$ encontraram em seu estudo sobre 
elaboração de pão utilizando farinha mista de trigo e linhaça menor firmeza para o pão-controle

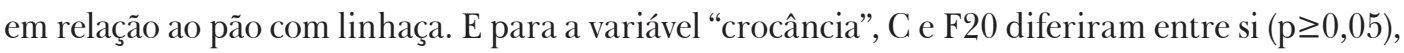
no entanto, F5 não apresentou diferença significativa dos demais ao nível de $5 \%$ (tabela 4).

Tabela 4. Avaliações físicas de biscoitos elaborados com farinha de trigo e com farelo de linhaça marrom em diferentes proporções. Pelotas, RS, 2012.

\begin{tabular}{lccc}
\hline Parâmetros & \multicolumn{3}{c}{ Tipos de Biscoitos } \\
\cline { 2 - 4 } Físicos & C & F5 & F20 \\
\hline MA (g) & $170,40 \pm 8,3^{\mathrm{a}}$ & $169,33 \pm 11,44^{\mathrm{a}}$ & $171,45 \pm 19,07^{\mathrm{a}}$ \\
MD (g) & $113,40 \pm 6,4^{\mathrm{a}}$ & $111,54 \pm 7,5^{\mathrm{a}}$ & $116,89 \pm 14,6^{\mathrm{a}}$ \\
FE (g) & $4,04 \pm 0,37^{\mathrm{b}}$ & $3,98 \pm 0,41^{\mathrm{b}}$ & $5,60 \pm 0,66^{\mathrm{a}}$ \\
VE (cm3. g-1) & $1,28 \pm 0,08^{\mathrm{a}}$ & $1,24 \pm 0,35^{\mathrm{a}}$ & $1,1 \pm 0,2^{\mathrm{a}}$ \\
CRC & $38,94 \pm 0,28^{\mathrm{a}}$ & $38,30 \pm 1,02^{\mathrm{a}, \mathrm{b}}$ & $37,73 \pm 0,54^{\mathrm{b}}$ \\
FMZ & $11463,5 \pm 522,4^{\mathrm{a}}$ & $8500,26 \pm 625,08^{\mathrm{b}}$ & $11357,51 \pm 1009,1^{\mathrm{a}}$ \\
\hline
\end{tabular}

* Médias seguidas de letras diferentes nas colunas diferem entre si pelo teste de Tukey $(p<0,05)$. MA: massa antes do forneamento; MD: massa depois do forneamento; FE: fator de expansão; VE: volume específico; CRC: crocância; FMZ firmeza.

** C: controle (trigo); F5: farelo de linhaça 5\%; F20: farelo de linhaça $20 \%$.

Os resultados dos atributos "sabor", "textura", "cor", "aparência”, "aroma” e "impressão global”, e respectivos desvios-padrão, estão apresentados na tabela 5. Observa-se diferença significativa $(\mathrm{p}<0,05)$ nos quesitos "sabor" e "impressão global". Em relação ao sabor, todas as médias apresentaram bom grau de aceitação. A amostra F5 obteve o maior valor de aceitação $(6,65)$, não havendo diferença significativa em relação ao F20. No entanto, os biscoitos elaborados com farinha de trigo apresentaram índice menor de aceitação (tabela 5), com diferença significativa $(\mathrm{p}<0,05)$. Resultados semelhantes foram relatados por Maciel et al., ${ }^{11}$ em estudo de biscoitos tipo cracker com adição da farinha de linhaça.

No quesito "impressão global" houve diferença significativa: o biscoito elaborado com a adição de farelo $5 \%$ superou as demais formulações, mas a formulação F20 não diferiu significativamente $(\mathrm{p}<0,05)$ da formulação controle (tabela 5). 
Tabela 5. Atributos sensoriais de biscoitos elaborados com farinha de trigo e com farelo de linhaça marrom em diferentes proporções. Pelotas. RS. 2012.

\begin{tabular}{cccc}
\hline \multirow{2}{*}{ Atributos Sensoriais } & \multicolumn{3}{c}{ Tipos de Biscoitos } \\
\cline { 2 - 4 } & $\mathrm{C}$ & $\mathrm{F} 5$ & $\mathrm{~F} 20$ \\
\hline Sabor & $5,92 \pm 1,80^{\mathrm{b}}$ & $6,65 \pm 1,75^{\mathrm{a}}$ & $6,05 \pm 2,11^{\mathrm{a}, \mathrm{b}}$ \\
Textura & $5,18 \pm 2,06^{\mathrm{a}}$ & $6,12 \pm 1,89^{\mathrm{a}}$ & $5,63 \pm 2,25^{\mathrm{a}}$ \\
Impressão Global & $6,20 \pm 1,57^{\mathrm{a}, \mathrm{b}}$ & $6,80 \pm 1,42^{\mathrm{a}}$ & $6,08 \pm 1,96^{\mathrm{b}}$ \\
Cor & $6,63 \pm 1,57^{\mathrm{a}}$ & $6,72 \pm 1,56^{\mathrm{a}}$ & $5,98 \pm 2,14^{\mathrm{a}}$ \\
Aparência & $6,82 \pm 1,46^{\mathrm{a}}$ & $6,72 \pm 1,02^{\mathrm{a}}$ & $6,02 \pm 1,46^{\mathrm{a}}$ \\
Aroma & $6,67 \pm 1,16^{\mathrm{a}}$ & $6,47 \pm 1,47^{\mathrm{a}}$ & $6,08 \pm 1,76^{\mathrm{a}}$ \\
\hline
\end{tabular}

**Letras diferentes na mesma coluna, para o mesmo nutriente, indicam diferença significativa pelo teste de Tukey $(0,05)$.

*** C: controle (trigo); F5: farelo de linhaça 5\%; F20: farelo de linhaça $20 \%$.

Os resultados obtidos na análise sensorial demonstraram que biscoitos com adição de farelo de linhaça, podem ser viáveis comercialmente, pois apresentam boa aceitabilidade e aumento da qualidade nutricional. Este ingrediente pode, de acordo com Borges et al.., ${ }^{20}$ Maciel et al. ${ }^{11} \mathrm{e}$ Oliveira et al. ${ }^{18}$, melhorar a palatabilidade do biscoito, tornando-o mais aceito pelos consumidores, resultados semelhantes aos encontrados no presente estudo.

\section{Conclusão}

A adição de farelo de linhaça a farinha de trigo enriqueceu a formulação do biscoito, aumentando seu valor nutricional, elevou o teor de proteínas, fibras e minerais. A formulação com adição de $5 \%$ de farelo de linhaça foi a mais aceita, mostrando ser viável e mais nutritiva para a alimentação diária. 


\section{Referências}

1. Wildman REC. Nutraceuticals: a brief review of historical and teleological aspects. In: Wildman REC. Handbook of nutraceuticals and functional foods. Boca Raton: CRC; 2001. p. 1-12.

2. Cui WS. Flaxseed: a functional food for the 21 st century. Canad. Chem. News.1998; 50(5):19-20.

3. Morris DH. Essential nutrients and other functional compounds in flaxseed. Nutr. Today. 2001; 36(3):159-162.

4. Marques AC. Propriedades funcionais da linhaça (Linum usitatissimum L.) em diferentes condições de preparo e de uso em alimentos. [Dissertação] Santa Maria: Universidade Federal de Santa Maria; 2008.

5. Figuerola F, Muñoz O, Estévez AM. La linaza como fuente de compuestos bioactivos para la elaboración de alimentos. Agr. Sur. 2008; 36(2):49-58.

6. Turatti JM. Óleos vegetais como fonte de alimentos funcionais. Óleos \& Grãos. 2000; 56:20-7.

7. Yuan YV, Rickard SE, Thompson LU. Short-term feeding of flaxseed or its lignan has minor influence on in vivo hepatic antioxidant status in young rats. Nutr. Res. 1999; 19(8):1233-1243.

8. Prasad K. Dietary flax seed in prevention of hypercholesterolemic atherosclerosis. Atherosclerosis. 1997; 132(1):69-76.

9. Hussain S, Anjum FM, Butt MS, Khan MI, Asghar A. Physical and sensoric attributes of flaxseed flour supplemented cookies. Turk J. Biol. 2006; 30:87-92.

10. Araújo JMA. Química de alimentos: teoria e prática. $3^{a}$ ed. Viçosa: UFV; 2004.

11. Maciel LMB, Pontes DF, Rodrigues MCP. Efeito da adição de farinha de linhaça no processamento de biscoito tipo cracker. Alim. Nutr. 2008; 19(4):385-392.

12. Association of Official Analytical Chemists. Official methods of analysis of the Association of Official Analytical Chemists. 16 ed. Arlington, VA: AOAC; 1995.

13. Bligh EG, Dyer WJ. A rapid method of total lipid extraction and purification. Can. J. Biochem. Physiol. 1959; 37(8): 911-917.

14. Carvalho CL, Mantovani DMB, Carvalho PRN, Moraes RM. Análises químicas de alimentos. Campinas: Instituto de Tecnologia de Alimentos; 1990. 121 p. Manual técnico.

15. Minim VPR. Análise sensorial: estudos com consumidores. Viçosa: Editora UFV; 2006.

16. Comissão Nacional de Normas e Padrões para Alimentos. Resolução 12/78 - Alimentos e bebidas: 47 padrões de identidade e qualidade. In: Associação Brasileira das Indústrias de Alimentação. Compêndio de resoluções da CNNPA. São Paulo: ABIA; 1978.

17. Teixeira E, Meinert EM, Barbetta PA. Análise sensorial de alimentos. Florianópolis: Editora UFSC; 1987.

18. Oliveira TM, Pirozi MR, Borges JTS. Elaboração de pão de sal utilizando farinha mista de trigo e linhaça. Alim. Nutr. 2007; 18(2):141-150. 
19. Heinemann RB, Costa NMB, Cruz R, Pirozi MR. Valor nutricional da farinha de trigo combinada com concentrado protéico de folha de mandioca. Rev. Nutr. 1998; 11(1):51-57.

20. Borges JTS, Pirozi MR, Paula CD, Ramos DL, Chaves JBP. Caracterização físico-química e sensorial de pão de sal enriquecido com farinha integral de linhaça. B. CEPPA. 2011; 29(1):83-96.

21. Maciel LMB. Utilização de farinha de linhaça (Linum usitatissimum L.) no processamento de biscoito tipo "cracker": características físico-químicas, nutricionais e sensoriais [Dissertação]. Fortaleza: Universidade Federal do Ceará; 2006.

22. Alpaslan M, Hayta M. The effects of flaxseed, soy and corn flours on the textural and sensory properties of a bakery product. J. Food Quality. 2006; 29(6):617-627.

23. Assis LM, Zavareze ER, Radünz AL, Dias ARG, Gutkoski LC, Elias MC. Propriedades nutricionais, tecnológicas e sensoriais de biscoitos com substituição de farinha de trigo por farinha de aveia ou farinha de arroz parboilizado. Alim. Nutr. 2009; 20(1):15-24.

24. Esteller MS, Lannes SCS. Parâmetros complementares para fixação de identidade e qualidade de produtos panificados. Cienc. Tec. Alim. 2005; 25(4):802-806. 
